\title{
Impairment of transneuronal traffic in Streptozotocin-induced diabetes, a WGA-HRP neurohistochemical study in the rat
}

\author{
Abayomi Odekunle \\ Anatomy Unit, Department of Basic Health Sciences, Faculty of Medical Sciences, The University of the West \\ Indies, St. Augustine, Trinidad and Tobago, West Indies \\ Received 6 February 2006; accepted 12 June 2006 \\ (C) 2006 National Science Council, Taipei
}

Key words: chronic streptozotocin-induced diabetes, stomach, wheat germ agglutinin-horseradish peroxidase, vagus nerve, retrograde transport, DMNV

\begin{abstract}
Retrograde transport of Wheat germ agglutinin conjugated to Horseradish peroxidase (WGA-HRP) was used in labeling vagal neurons projecting to the stomach from the dorsal motor nucleus of the vagus nerve (DMNV) in Streptozotocin (STZ)-induced diabetic rats. Diabetes was induced in the experimental rats by intraperitoneal injection of buffered STZ. Control rats were injected with an equivalent volume of the citrate buffer not containing STZ.The experimental rats, which became diabetic about $24 \mathrm{~h}$ after intraperitoneal injection of STZ, were kept alive for a period of 24 weeks to attain a chronic state of diabetes. Control euglycaemic rats were also kept alive for 24 weeks. At the end of 24 weeks, the two groups of rats were prepared for stomach surgery. Following anaesthesia laparotomy was performed and the stomach exteriorized. The anterior and posterior walls of the stomach were injected with $0.1 \mathrm{ml}$ of $5 \% \mathrm{WGA}-\mathrm{HRP}$ in $0.5 \mathrm{M}$ sodium chloride. Experimental and control rats were sacrificed $48-72 \mathrm{~h}$ after tracer injection by transcardial perfusion with normal saline, fixative and buffered sucrose.Transverse serial frozen sections of the brainstem were processed for WGA-HRP neurohistochemistry and analyzed under light and dark-field microscopy. The analyses of the sections taken from the chronic diabetic rats revealed fewer WGA-HRP labeled neurons in the DMNV than sections taken from the control euglycaemic rats. The depletion of labeled neurons in the diabetic rats compared with the euglycaemic rats is indicative of an interference with the mechanism of retrograde neuronal transport of WGA-HRP by chronic diabetic state.
\end{abstract}

\section{Introduction}

Diabetic autonomic peripheral neuropathy is a fairly common and difficult-to-manage late complication of diabetes. The gastrointestinal system is one of the commonly affected systems where the vagus nerve usually shows signs of demyelination [1-3] and degeneration of myelinated fibres, axonal terminals and dendrites [2-6]. Gastric dysfunctions (diabetic gastropathies) associated with diabetic vagal lesion

*To whom correspondence should be addressed. E-mail: Odekunle@tstt.net.tt range from, tachygastria, antral hypomotility, and frank gastroparesis to abnormal myoelectric activities. These gastropathies manifest in form of abdominal discomfort, nausea, vomiting, nocturnal diarrhea, postprandial fullness, and early satiety [7]. Earlier studies have shown that vagal lesions in chronic STZ-induced diabetes were caused by hyperglyceamia of diabetes $[8,9]$ and are associated with increased amount of metabolic markers which include glucose, fructose and sorbitol in the vagus nerve [8]. Vagal lesions of chronic diabetes have also been shown to be accompanied by impairment in the neuronal transport capabilities of the vagus nerve. 
$[8,10,11,12]$. Lee et al. have demonstrated impairment in the ability of the vagus nerve to transport target tissue-derived endogenous material such as nerve growth factor (NGF) and neurotrophin-3 (NT-3) in diabetic rat but reported no change in the content of these substances in the vagally innervated organs studied [11]. These investigators went on to suggest that diminished access to target-derived neurotrophin could not have been responsible for the diabetic-induced alteration in the retrograde axonal transport of neurotrophin. Following these findings, investigators have hypothesized that neuronal functional abnormalities such as observed in the diabetic state are accompanied by disruptions in the mechanism of neuronal transport in the affected vagus nerve. This hypothesis is supported by the demonstration of impairment of anterograde and retrograde transport of NGF, NT-3 and the neuronal tracer fluorogold in the vagus nerve of STZ-induced chronic diabetic states $[8,10,11,12]$.

It has therefore been inferred that the reduced transport of trophic factors is due to impaired axoplasmic transport and further impacts upon the integrity of the cell bodies of origin of vagal nerve fibres. While reduced neuronal transport in the diabetic rat could be due to impairment of transport mechanism, it could also emanate from loss of vagal neurons or glial cell dysfunction as a direct metabolic effect of hyperglycaemia. The present study was designed to determine the effect of STZ-induced diabetes on the uptake and retrograde transport of a sensitive neuronal tracer WGA-HRP by gastric vagal nerve fibres in the rat.

\section{Materials and methods}

Adult male and female Sprague-Dawley rats weight range between 200 and 300 gm were used for the study. These were divided into two groups (Experimental and Control). The experimental rats were injected intraperitoneally with STZ in a dosage of $60 \mathrm{mg} / \mathrm{kg}$ in $0.05 \mathrm{M}$ sodium citrate buffer at $\mathrm{pH} 4.5$ after light anaesthesia with ketamine/xylazine. Twentyfour hours after STZ injection blood glucose levels were measured from tail-prick blood sample to confirm induction of hyperglycaemia. The diabetic rats were subsequently treated with lent insulin at a daily dosage of 2-3 IU/100 g body weight to maintain a steady level of blood glucose between 200-300 gm/dl for a period of 24 weeks. Experimental rats' blood glucose level was monitored on a weekly basis throughout the duration of the experiment. Control rats were injected in the same manner as the experimental rat with the same volume of 0.05 $\mathrm{M}$ citrate phosphate buffer, $\mathrm{pH}$ 4.5. Blood glucose level of control rats was also measured $24 \mathrm{~h}$ after the injection to confirm a euglycaemic level of $<200 \mathrm{mg} / \mathrm{dl}$. This was also measured weekly throughout the duration of the experiment to ascertain euglycaemic status of control rats.

At the end of 24 weeks, experimental and control rats, which survived this phase of the experiment, were selected for treatment with WGA-HRP.

For surgical exposure of the stomach, each rat was anaesthetized with an intramuscular injection of $40 \mathrm{mg} / \mathrm{kg}$ ketamine and $8 \mathrm{mg} / \mathrm{kg}$ of xylazine. A midline laparotomy was then performed and the stomach was delivered to the anterior abdominal wall.

With the aid of a $100 \mu 1$ Hamilton syringe with a G31 gauge needle, $0.1 \mathrm{ml}$ of 5\% WGA-HRP in $0.5 \mathrm{M}$ sodium chloride was injected by multiple penetrations into the muscular coat of the anterior and posterior walls of the stomach. A total of 10 penetrations were made in the central area of the body of the stomach (5 into the anterior surface and 5 into the posterior surface)-Figure 1. The injection site was maintained consistently for each experiment. In order to minimize leakage of tracer into the peritoneal cavity, the injecting needle was left in place for about 2 min after each penetration. On withdrawal of the needle, the injection site was further pressed firmly with sterile gauze for $2 \mathrm{~min}$ to reduce tracer leakage. After the injections the stomach was wiped with sterile gauze and returned to the peritoneal cavity. The abdominal incision was then closed in layers and the rats allowed to recover from anaesthesia in a warm environment.

Experimental and control rats were sacrificed $72 \mathrm{~h}$ after tracer injection by anaesthesia which was followed by transcardial perfusion first with normal saline followed by a fixative containing $1 \%$ paraformaldehyde and $1.25 \%$ glutaraldehyde in $0.1 \mathrm{M}$ phosphate buffer, $\mathrm{pH} 7.4$ at room temperature and finally with $10 \%$ buffered sucrose at $4^{\circ} \mathrm{C}$.

Transverse serial frozen sections of the brainstem, $40 \mu$ in thickness were then taken and 


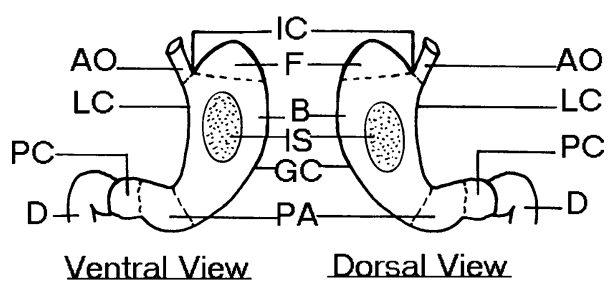

Figure 1. Schematic representation of the stomach and adjoining segments of the digestive tract illustrating the injection site (dotted areas) of WGA-HRP into the anterior and posterior walls of the stomach. $\mathbf{A O}=$ Abdominal oesophagus; $\mathbf{I C}=$ Incisura cardiaca; $\mathbf{F}=$ Fundus; $\mathbf{L C}=$ Lesser curvature; $\mathbf{B}=$ Body of the stomach; $\mathbf{I S}=$ Injection site; $\mathbf{G C}=$ Greater curvature; $\mathbf{P A}=$ Pyloric antrum; $\mathbf{P C}=$ Pyloric canal; $\mathbf{D}=$ Duodenum .

processed for WGA-HRP neurohistochemistry, using the modified tetramethyl benzedene (TMB) procedure recommended by Mesulam [13]. The sections were then analyzed under light and darkfield microscopy. For cell counting, cells with distinct margins and in which the blue-black reaction product of TMB/WGA-HRP were seen in their cytoplasm were regarded as WGA-HRP labeled cells. Counting of labeled cell on either side of the midline was done from every other section with the aid of a calibrated cell counting chamber. This selection of sections for counting was done to avoid counting the same cell twice.

\section{Results}

Analysis of sections taken from the brainstem of diabetic and non-diabetic rats $72 \mathrm{~h}$ after injection of WGA-HRP revealed the presence of WGAHRP labeled neurons in DMNV. Labeled neurons were bilaterally distributed in both groups of rats. Furthermore, whereas labeled neurons were seen along the entire rostrocaudal extent of the DMNV, they were restricted to the medial aspect along the mediolateral axis of the nucleus in both groups of rats (Figures 2-4). The numbers of labeled neurons counted in the DMNV of both side in the diabetic rats were also found to be significantly less than the number of labeled neurons counted in the non-diabetic rats, $p<0.05$ (Tables 1 and 2). The average rostrocaudal extent of labeling of the DMNV was from $1.8 \mathrm{~mm}$ caudal to the obex to $4.4 \mathrm{~mm}$ rostral to the obex. An average of 155 sections containing labeled cells was obtained per animal. On the average, about 70 sections were actually used for cell counting. It must however be noted that the density of cell labeling was not uniform throughout the rostrocaudal extent of the labeled region

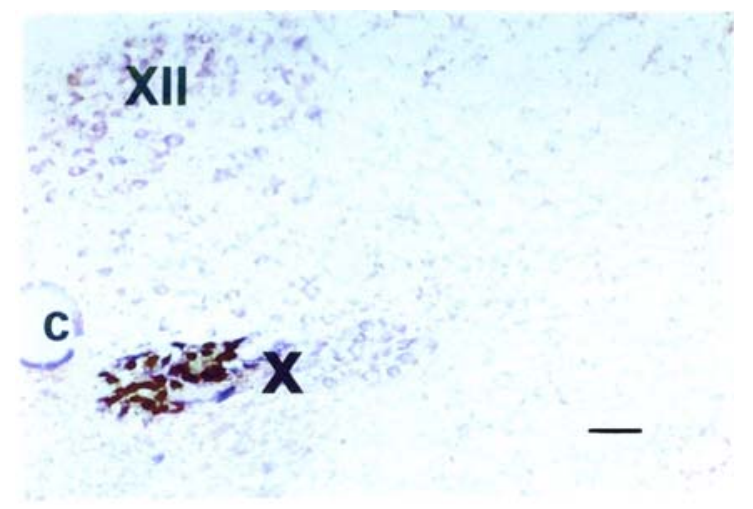

Figure 2. Photomicrograph of a representative transverse section taken from the brainstem of a STZ-induced diabetic rat demonstrating WGA-HRP labeled neurons (Colored black) in the dorsal motor nucleus of the vagus nerve following injection of the tracer into the ventral and dorsal walls of the stomach. Note the reduction in the population of labeled neurons in this micrograph compared with Figure 3. C. Central canal, closed part of the Medulla oblongata; $\mathbf{X}$. Dorsal motor nucleus of the vagus nerve; XII. Hypoglossal nucleus; Scale Bar $=25 \mu$.

(1.8 mm caudal to the obex to $4.4 \mathrm{~mm}$ rostral to the obex). Labeling was most intense in the region of the obex but diminished rostrally and caudally.

No other vagal brainstem nuclei were labeled in the study.

\section{Discussion}

The retrograde labeling of neurons in the DMNV following injections of WGA-HRP into the ventral and dorsal walls of the stomach in the diabetic and non-diabetic rats in the present study was anticipated since earlier studies had identified the DMNV as the principal source of vagal fibres innervating the stomach in most animal species [14-17]. It is however quite interesting that labeled neurons in the diabetic rats were significantly 


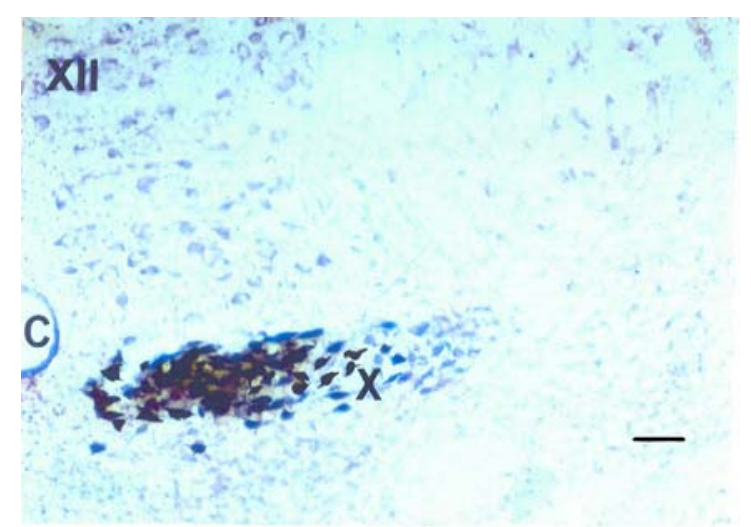

Figure 3. Photomicrograph of a representative transverse section taken from the brainstem of a non-diabetic (control) rat demonstrating WGA-HRP labeled neurons (colored black) in the dorsal motor nucleus of the vagus nerve following injection of the tracer into the ventral and dorsal walls of the stomach. C. Central canal, closed part of the Medulla oblongata; X. Dorsal motor nucleus of the vagus nerve; XII. Hypoglossal nucleus; Scale Bar $=25 \mu$.

A
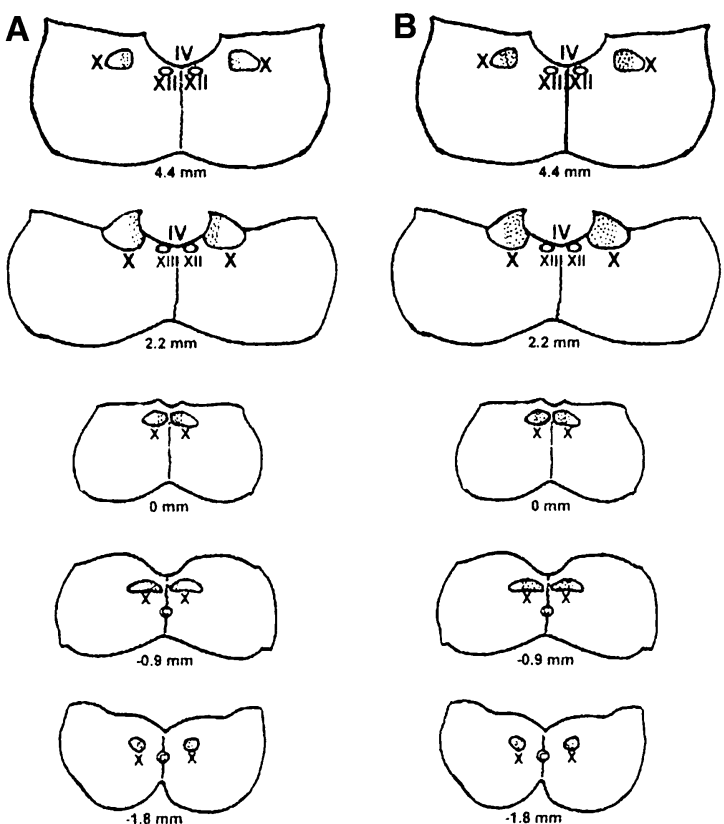

Figure 4. Schematic representations of transverse sections of the brainstem demonstrating the distribution of WGAHRP-labeled neurons (dotted areas) in the DMNV following injections of the tracer into anterior and posterior walls of diabetic (A) and non-diabetic (B) rats. Representative sections were taken from levels $4.4 \mathrm{~mm}$ and $2.2 \mathrm{~mm}$ rostral to the obex, the obex (level $0 \mathrm{~mm}$ ) and levels $0.9 \mathrm{~mm}$ and $1.8 \mathrm{~mm}$ caudal to the obex. IV. Fourth ventricle; X. Dorsal motor nucleus of the vagus nerve (DMNV); XII. Hypoglossal nucleus C. Central canal of the closed part of the medulla oblongata.
Table 1. Table of data of WGA-HRP labeled cell counts following injection of the tracer into the muscular coat of the stomach of diabetic rats.

\begin{tabular}{lc}
\hline $\begin{array}{l}\text { Code number of } \\
\text { experimental rats }\end{array}$ & $\begin{array}{l}\text { Number of labeled neurons } \\
\text { counted in diabetic rats }\end{array}$ \\
\hline D1 & 396 \\
D2 & 420 \\
D3 & 427 \\
D4 & 398 \\
D5 & 415 \\
D6 & 410 \\
Mean & 411 \\
SEM & \pm 4.993 \\
\hline
\end{tabular}

Table 2. Table of data of WGA-HRP labeled cell counts following injection of the tracer into the muscular coat of the stomach of non-diabetic (control) rats.

\begin{tabular}{lc}
\hline $\begin{array}{l}\text { Code number of } \\
\text { experimental rats }\end{array}$ & $\begin{array}{l}\text { Number of labeled neurons } \\
\text { counted in non-diabetic rats }\end{array}$ \\
\hline ND1 & 845 \\
ND2 & 794 \\
ND3 & 856 \\
ND4 & 902 \\
ND5 & 798 \\
ND6 & 840 \\
Mean & 839 \\
SEM & \pm 16.331 \\
\hline
\end{tabular}

reduced compared with labeled neurons in the non-diabetic rats. The present result is quite consistent with those of Lee et al. in which significant reduction of fluorogold-labeled neurons were observed in STZ-induced diabetic rats compared with non-diabetic control rats following injections of a different tracer, fluorogold into regions of the stomach. It is also noteworthy that both studies in which different tracers were used in the same organ labeled neurons in the medial aspect of the DMNV.

Several neurotrophic mechanisms and axonal transport system of the neuron are implicated in the transport of neuronal tracer by neurons. Derangement of any of these systems by the metabolic disorder associated with chronic diabetes could have been responsible for the depletion of labeled vagal neurons observed in the diabetic rats in the present study. The observed depletion of labeling in this and previous studies could also have been due to a reduction in the availability of 
the injected tracer to the nerve terminal as a result of degeneration of vagal efferent terminals or gastric neuropathy such as gastric hypertrophy associated with the metabolic derangement of diabetes. On the other hand, diabetic-induced impairment of uptake of the tracer through the processes of endocytosis and internalization could have been responsible for the impairment of retrograde transport of tracer observed in the present study.

Electron microscopic study has also demonstrated degenerative changes in the dorsal motor nucleus of the vagus nerve in diabetic rat [9]. Thus direct neuronal loss can not be ruled out as the likely cause of reduction in the labeled neurons in the present study.

The result of the present study is also consistent with those in which endogenous neurotrophins such as nerve growth factor and neurotrophin-3 which are also transported by the same neural traffic system have been shown to be similarly affected in STZ-induced chronic diabetic states [11]. The failure to label any neurons in the nucleus ambiguous in the present study is consistent with earlier studies which have shown that the parts of the stomach injected in the present study are innervated only by the DMNV [18-21]. In an earlier report [11] weak labeling of the nucleus ambiguous (nA) were observed. This was however subsequent to tracer injection into the regions of the stomach reported to contain "fibres of passage" originating partly from the nA [18-20, 22].

In concusion the present study has provided evidence of impaired neuronal transport in the vagal nerve in diabetic rat. We have also suggested underlying mechanisms of the impaired neuronal function. Further investigations at the peripheral and central ends of the vagal system are required to determine the precise mechanisms of the defective neuronal functions reported in this and previous related studies.

Acknowledgements This study was jointly supported by The Campus Research and Publication Fund Committee, University of the West Indies, St. Augustine Campus and The Caribbean Health Research Council. The technical assistance offered by Ms N. Homer and Ms C. Lord in the Research Laboratory is greatly appreciated.

\section{References}

1. Britland S.T., Young R.J., Sharma A.K., Lee D., Ah-See A.K. and Clarke B.F., Vagus nerve morphology in diabetic gastropathy. Diabet. Med. 7: 780-787, 1990.

2. Duchen L.W., Anjori A., Watkins P.J. and Mackay J.D., Pathology of autonomic neuropathy in diabetes mellitus. Ann. Intern. Med. 92: 301-303, 1980.

3. Kristensson K., Nordborg C., Olsson Y. and Sourander P., Changes in the vagus nerve in diabetes mellitus. Acta. Pathol. Microbiol. Scand. (A) 79: 684-685, 1971.

4. Guo Y.P., Mcleod J.G. and Baverstock J., Pathological changes in the vagus nerve in diabetes and chronic alcoholism. J. Neurol. Neurosurg. Psychiatr. 50: 14491453, 1987.

5. Guy R.J., Dawson J.L., Garrett J.R., Law J.W., Thomas P.K., Sharma A.K. and Watkins P.J., Diabetic gastroparesis from autonomic neuropathy: surgical considerations and changes in vagus morphology. J. Neurol. Neursurg. Psychiatr. 1984(47) 686-691, 1984.

6. Schmidt R.E., Modert C.W., Yip H.K. and Johnson E.M. Jr., Retrograde axonal transport of intravenously administered 125I-nerve growth factor in rats with streptozotocininduced diabetes. Diabetes 32(7) 654-663, 1983.

7. Koch K.T., Diabetic gastropathy: gastric neuromuscular dysfunction in diabetes mellitus: a review of symptoms, pathophysiology and treatment. Dig. Dis. Sci. 44(6) 10611075, 1999.

8. Lee P.G., Thomas C.H., Cai F., Regalia J. and Helke C.J., Streptozotocin-induced diabetes causes metabolic changes and alterations in neurotrophins content and retrograde transport in the cervical vagus nerve. Exp. Neurol. 170: 149-161, 2001

9. Tay S.S. and Wong W.C., Short- and long-term effects of STZ-induced diabetes on the dorsal motor nucleus of the vagus nerve in the rat. Acta Anat. (Basel) 150: 274-281, 1994.

10. Cai F. and Helke C.J., Abnormal PI3 kinase/AKt signal pathway in vagal afferent neurons and vagus nerve of streptozotocin-diabetic rats. Brain Res. Mol. Brain Res. 110(2) 234-244, 2003.

11. Lee P.G., Cai F. and Helke C.J., Streptozotocin-induced diabetes reduces retrograde axonal transport in the afferent and efferent vagus nerve. Brain Res. 941(1-2) 127-136, 2002.

12. Hellweg R. and Hartung H.D., Endogenous levels of nerve growth factor (NGF) are altered in experimental diabetes mellitus: a possible role for NGF in the pathogenesis of diabetic neuropathy. J. Neurosci. Res. 26: 258-267, 1990.

13. Mesulam M.M., Tracing neural connections with horseradish Peroxidase. A Wiley-Interscience Publication, New York, 1982, pp. 1-152.

14. Brtva R.D., Iwamoto G.A. and Longhurst J.C., Distribution of cell bodies for primary afferent fibres from the stomach of the cat. Neurosci. Lett. 105: 287-293, 1989.

15. Loewy A.D. and Spyer K.M., Vagal Preganglionic Neuron: Central regulation of autonomic function. Oxford University Press, New York, 1990, pp. 68-87.

16. Neuhuber W.L., Sensory vagal innervation of the rat esophagus and cardia: a light and electron microscopic anterograde tracing study. J. Auton. Nerv. Syst. 20: 243255, 1987.

17. Shapiro R.E. and Miselis R.R., The central organization of the vagus nerve innervating the stomach of the rat. J. Comp. Neurol. 238: 473-488, 1985. 
18. Takayama K., Ishikawa N. and Miura M., Sites of origin and termination of gastric vagus Preganglionic neurons: An HRP study in the rat. J. Auton. Nerv. Syst. 6: 211-23, 1982.

19. Jou M.J., Wen C.Y. and Shieh J.Y., Localization of the stomach-projecting neurons in the dorsal motor nucleus of the vagus nerve in the guinea pig. J. Auton. Nerv. Syst. 43: 201-6, 1993.

20. Odekunle A. and Chinnah T.I., Brainstem origins of gastric vagal preganglionic parasympathetic neurons and topographic representation of the stomach in the dorsal motor nucleus of the vagus nerve: an HRP study in the ferret. West Afr. J. Anat. 6: 1-8, 1998.
21. Iizuka Y. and Sugita S., Vagal preganglionic neurons innervating the different gastric regions in the Japanese quail (Coturnix japonica). Okajima Folia Anatomy. Jpn. 72(2-3) 149-162, 1995.

22. Odekunle A., Adogwa A. and Senok S.S., Evidence of collateralization of vagal efferents innervating subdiaphragmatic segments of the gastrointestinal tract in the rat using the double labeling fluorescent dyes technique. West Indian Med J 51(4) 216-19, 2002. 